\title{
Effect of Some Non steroidal Anti-Inflammatory Drugs on Growth, Adherence and Mature Biofilms of Candida
}

\section{spp.}

\author{
Ahmad Ashraf ${ }^{1}$, Fatma Yousri ${ }^{1}$, Nora Taha ${ }^{1}$, Omar Abd El-Waly ${ }^{2}$, Abd El-Kareem Ramadan ${ }^{2}$, Esraa Ismail ${ }^{2}$, \\ Reham Hamada ${ }^{1}$, Mohamed Khalaf ${ }^{1}$, Mohamed Refaee ${ }^{1}$, Sameh $\mathrm{Ali}^{3}$, Abobakr Madyn ${ }^{4}$, \\ Rehab Mahmoud Abd El -Baky,** \\ ${ }^{1}$ Undergraduate student, faculty of pharmacy, Minia university \\ ${ }^{2}$ Undergraduate student, Faculty of science, Minia university \\ ${ }^{3}$ Demonstrator, Department of Microbiology, Faculty of Pharmacy, Minia university \\ ${ }^{4}$ A researcher, Department of Microbiology, Faculty of Pharmacy, Minia university \\ ${ }^{5}$ Lecturer of Microbiology, Department of Microbiology, Faculty of Pharmacy, Minia university \\ *Corresponding author: dr_rehab010@yahoo.com
}

Received December 05, 2014; Revised December 25, 2014; Accepted January 05, 2015

\begin{abstract}
Candida spp. are the most common cause of fungal diseases and the fourth commonest cause of nosocomial invasive infections which are considered in many cases as life threatening. Among Candida spp., $C$. albicans is the most common cause of many fungal diseases, but non-albicans spp. infections are in increase. Nonsteroidal anti-inflammatory drugs have previously been shown to have antifungal activity. In this study we determine the antifungal activity of the tested NSAIDs using agar well diffusion method, their effect on the dimorphic transition of C. albicans by testing their ability to form germ tube in the presence of human serum. Determining the effect of NSAIDs on the adherence to plastic surfaces and on the mature biofilms formed by the tested Candida spp.. The results indicated that Sodium Diclofenac showed lower MIC against C. albicans and C. glabrata while Ibuprofen had lower MIC against C. krusei. upon examining the effect of Diclofenac sodium, Ibuprofen and ketoprofen on biofilm formed on polyurethane segments by Scanning electron microscope (SEM), a damage to membranes of the tested species was observed. Sodium Diclofenac showed the highest inhibitory effect on the adherence (51.1-76.9\% at MIC and 56.6-83.3\% at 2XMIC) of C. albicans and C. glabrata but Ibuprofen showed a higher inhibitory effect against the adherence of $C$. krusei. For mature biofilms, the highest disruptive effect on mature biofilms formed by all tested Candida Spp. (37.72-59.29\% at MIC and $42.68-63.06 \%$ at 2XMIC) was observed by Diclofenac sodium. Sodium Diclofenac inhibited dimorphic transition of C. albicans but a decrease in germ tube formation was shown by others. In conclusion, the tested drugs showed antifungal, anti-adherent and antibiofilm activity that make them useful in the treatment of fungal infection and the prevention of biofilm formation on the surface of medical devices.
\end{abstract}

Keywords: Ketoprofen, Ibuprofen, Diclofenac sodium, biofilm, Candida, Dimorphic transition

Cite This Article: Ahmad Ashraf, Fatma Yousri, Nora Taha, Omar Abd El-Waly, Abd El-Kareem Ramadan, Esraa Ismail, Reham Hamada, Mohamed Khalaf, Mohamed Refaee ${ }^{1}$, Sameh Ali, Abobakr Madyn, and Rehab Mahmoud Abd El-Baky, "Effect of Some Non steroidal Anti-Inflammatory Drugs on Growth, Adherence and Mature Biofilms of Candida spp.” American Journal of Microbiological Research, vol. 3, no. 1 (2015): 1-7. doi: 10.12691/ajmr-3-1-1.

\section{Introduction}

Most of microbes were found to be able to adhere and colonize biotic or abiotic surfaces. Candida cells, like many other microorganisms, are able to adhere to and colonize mucous membranes and surfaces of medical devices, resulting in the formation of a biofilm mass. Candida biofilms occurrence on medical device were proven upon the examination and the visualization of medical devices removed from patients or animal models using scanning electron microscopy, confocal laser scanning microscopy [1,2,3]. Fungal biofilm associated infections are considered as a major clinical concern as these structured microbial communities, embedded in an extracellular matrix, are characterized by increased resistance to host immune defense and antifungal therapy [4]. Biofilm resistance may be attributed to the presence of different mechanisms: (i) expression of efflux pumps, (ii) a changing sterol composition in the membrane, (iii) extracellular matrix as a barrier for the diffusion of antifungals and the molecules of immune system (iv) the presence of persisters (dormant cells not mutants) [5,6]. Medical devices can become colonized by one or more microorganisms which form a biofilm consisting of a 
mono- or multilayer cells embedded within an exopolysaccharide matrix [7-13]. Detachment of microorganisms from the biofilm may initiate an acute disseminated infection. Implant-associated infections are difficult to be treated increasing the need of implant removal $[8,12,14]$. Although the majority of implant infections are caused by bacteria, fungal biofilm associated infections tend to be more serious. Fungal infections are most commonly caused by the pathogenic Candida species, particularly C. albicans, C. tropicalis, and $C$. parapsilosis that are regarded as increasingly important nosocomial pathogens [15]. Candida bloodstream infections associated with intravascular catheters represented $40 \%$ of all nosocomial septicemias occurring in intensive care unit patients with a high mortality rate $[11,16,17]$. Also, Candida biofilms were found associated with urinary catheters, prosthetic heart valves, cardiac pacemakers, silicone voice prostheses, endotracheal tubes, and cerebrospinal fluid shunts [18].

Non steroidal anti-inflammatory drugs are widely used, especially as anti-inflammatory and analgesics. NSAIDs inhibit the cyclooxygenase isoenzymes COX-1 and COX2 , which are involved in the biosynthesis of mammalian prostaglandins. Prostaglandins are small lipid molecules showing different functions [19]. They are also found to be produced by $C$. albicans cells but their role in fungal biology is unknown [20]. Erb-Downward and Noverr [21] suggested that prostaglandins can act as regulators for $C$. albicans virulence. As eicosanoids pathway was reported to show a vital role in controlling yeast-hyphal transition and biofilm. So, drugs targeting fungal prostaglandins pathways may show great effects on fungal colonization and infection [21]. In addition, some studies showed that Sodium Diclofenac affect C. albicans morphogenesis and biofilm formation. [20,22]. Also, Ibuprofen show fungistatic activity at low concentrations and fungicidal activity at high concentrations [23]. In this study we tested the antifungal activity of three NSAIDs on different Candida Spp. and their effect on biofilm formation

\section{Materials and Methods}

\subsection{Microbial Strains}

Three different species of Candida, C. albicans, C. glabrata, C. Krusei obtained from the department of microbiology and immunology, Faculty of medicine, Assuit university were used in this study. The identification of organisms were based on the following: colony morphology, germ tube test, chlamydospores on Tween 80 cornmeal agar (Difco) and by the pattern of assimilation of a variety of carbon and nitrogen sources [24]. C. albicans ATCC 10231 was obtained from MIRCIN culture collection of the Faculty of Agriculture, Ain Shams University, Egypt.

\subsection{Drugs}

The following drugs were used Sodium Diclofenac (Glaxo, Egypt), Ibuprofen (Kahira/Abbott, Egypt) and Ketoprofen (Amriya, Egypt).Stock solutions were prepared as shown in Table 1. Working solution concentrations were ranged from (20-0.078125 mg/ml).

Table 1. Preparation of NSAIDs stock solution

\begin{tabular}{|c|c|c|c|}
\hline Drug & Stock solution conc. & Drug (mg) & Solvent (ml) \\
\hline Sodium Diclofenac & $20 \mathrm{mg} / \mathrm{ml}$ & Water (100 ml) & 2 gm \\
\hline Ketoprofen & $20 \mathrm{mg} / \mathrm{ml}$ & Water (100 ml) & 2 gm \\
\hline Ibuprofen & $20 \mathrm{mg} / \mathrm{ml}$ & DMSO (100 ml) \\
\hline
\end{tabular}

\subsection{Detection of Biofilm Formation by Tissue Culture Plate Method (TCP)}

The TCP assay is most widely used and was considered as standard test for detection of biofilm formation. All isolates were screened for their ability to form biofilm by the TCP method as described by Christensen et al. [25] with a modification in duration of incubation which was extended to 24 hours, according to O'Toole and Kolter [26].

\subsection{Evaluation of Antifungal Activity of the tested NSAIDs Using well Diffusion Technique}

Candida spp. (0.5 ml) of $1 \times 10^{8} \mathrm{CFU} / \mathrm{ml}$ (0.5 Mcfarland turbidity) were plated in sterile petri dishes then $20 \mathrm{ml}$ of sterile, molten and cooled $\left(45^{\circ} \mathrm{C}\right)$ Muller Hinton agar media with the addition of methylene blue-glucose (to enhance zone diameter visualization) was added to all petri dishes. The plates then were rotated slowly to ensure uniform distribution of the microorganisms and then allowed to solidify on a flat surface. After solidification, four equidistant and circular wells of $10 \mathrm{~mm}$ diameter were carefully punched using a sterile cork bore. Each sample was applied as triplicate. The plates were allowed to stand for one hour for prediffusion of the extract to occur then incubated overnight at $37^{\circ} \mathrm{C}$. All plates were examined and zones of inhibition were recorded $[27,28,29]$.

\subsection{Determination of Minimum inhibitory Concentrations (MIC) of the Tested NSAIDs:}

Two fold serial dilutions were performed on the tested NSAIDs. Equal volumes of each dilution were applied separately to each well in three replicates using a micropipette. All plates were incubated at $37^{\circ} \mathrm{C}$ for 24 hours, then the formed zones of inhibition were measured. The average of the zones of inhibition was calculated. The minimum inhibitory concentration (MIC) was calculated by plotting the natural logarithm of the concentration of each dilution of the tested NSAIDs against the square of zones of inhibition. A regression line was drawn through the points. The antilogarithm of the intercept on the logarithm of concentration axis gave the MIC value [29].

\subsection{Germ Tube Formation}

C. albicans strains were cultivated on Sabouraud's dextrose agar for one day. Cells were suspended in sterile saline and adjusted to density of 0.5 Mcfarland turbidity. $400 \mu \mathrm{l}$ of human serum was added to $1 \mathrm{ml}$ of cell 
suspensions of $C$. albicans. Drugs were added to the suspensions to a final concentration ranged from 100 to $1000 \mu \mathrm{g} / \mathrm{ml}$ and saline was added to the control tube. The cell suspensions were incubated with gentle shaking at $37^{\circ} \mathrm{C}$ for 2 hours and were examined for the presence of germ tubes by using a light microscope. Photomicrographs of colonies and invasive growth were taken with a DMRXA microscope (Leica, Germany) [30].

\subsection{Effect of Ketoprofen, Diclofenac Sodium, Ibuprofen on the Adherence of the Tested Candida spp. on Plastic Surfaces}

All strains were first streaked onto YEPD agar (1\% yeast extract, $2 \%$ Bacto peptone, 2\% D-glucose, 1.5\% agar) then, incubated at $25^{\circ} \mathrm{C}$ for 48 hours. A large loop of actively growing cells (for each strain) was transferred to sterile trypticase soy broth (TSB) (Difco Laboratories) containing $0.9 \%$ D-glucose. After incubation at $25^{\circ} \mathrm{C}$ for 24 hours, the cells were centrifuged and washed twice with $0.5 \mathrm{ml}$ PBS (phosphate buffered saline), followed by vortexing and centrifugation at $5000 \mathrm{~g}$ for $5 \mathrm{~min}$. The washed cells were suspended in $1 \mathrm{ml}$ TSB broth and adjusted to a final OD600 nm value of 1.0 with TSB broth. These cell suspensions were then used to grow biofilms.

$100 \mu \mathrm{l}$ of the suspension (OD600) was inoculated into individual wells of polystyrene 96 -well plates (flat bottom; Nunc). TSB broth was used as a negative control. The plates were incubated at $25^{\circ} \mathrm{C}$ for 90 min (adhesion period). Supernatants including planktonic cells were discarded and wells were gently washed with PBS twice to remove any non-adherent cells. $100 \mu \mathrm{l}$ of fresh TSB broth containing one of, MIC, above MIC and under the MIC concentrations of each of the following solutions: Ketoprofen, ibuprofen, and Sodium Diclofenac was added to each well. The plates were covered to prevent evaporation and incubated at $25^{\circ} \mathrm{C}$ for 24 hours. Liquid media containing the non adherent cells were discarded through two rounds of washing with $200 \mu$ l sterile PBS buffer. Adherent cells to the plastic surfaces were quantified using Crystal violet assay. Experiment was performed in triplicate.

\subsection{Effect of Ketoprofen, Diclofenac Sodium, Ibuprofen on the Preformed Biofilms of the Tested Candida spp. on Plastic Surfaces}

$100 \mu \mathrm{l}$ of the suspension (OD600) was inoculated into individual wells of polystyrene 96-well plates (flat bottom; Nunc). The plates were incubated at $25^{\circ} \mathrm{C}$ for 48 hours. After the incubation period, the supernatants from each well were aspirated and the wells washed twice with PBS without disturbing the biofilms at the bottom of the wells, then one of sub MIC, MIC, above MIC of each of the following solutions: Ketoprofen, ibuprofen, and Sodium Diclofenac was added to wells each alone. Normal saline without any agents was added to the control wells. The plates were incubated at $25^{\circ} \mathrm{C}$ for 24 hours. Supernatants were discarded through two rounds of washing with 200 $\mu \mathrm{l}$ sterile PBS buffer. Cells adherent to the plastic surfaces were quantified using Crystal violet assay [31]. Experiment was performed in triplicate.

\subsection{Scanning Electron Microscopy (SEM)}

The untreated Candida spp (control) and Candida spp. treated with the tested drugs were fixed in $2.5 \%$ ( $\mathrm{vol} / \mathrm{vol})$ glutaraldehyde in Dulbecco PBS (PH 7.2) for 1.5 hours, rinsed with PBS, and then dehydrated through an ethanol series. Samples were dried and gold-palladium coated. SEM examinations were made on a JSM-840 SEM (JEOL Ltd., Tokyo, Japan) [32].

\subsection{Statistical Analysis}

One-Way ANOVA as employed to evaluate any significant difference between the values obtained without the drug (controls) and the values obtained in the presence of different drug concentrations. Differences were done using SPSS version19 (SPSS Inc., Chicago, IL) for windows was used. Descriptive statistics were calculated. Kruskal-Wallis test was used to compare groups. A significant $P$-value was considered when it was less than 0.05 .

\section{Results}

\subsection{Biofilm Production}

Four Candida spp. were tested for their ability to form biofilm and according to TCP method strains can be classified into weak adherent at OD $<0.120$, moderate $0.120-0.240$ and strong $>0.240$ (Table 2)

Table 2. Biofilm production by Candida spp.

\begin{tabular}{|c|c|}
\hline Microorganisms & Ability to adhere to TCP surface \\
\hline C. albicans ATCC 10231 & Moderate \\
\hline C. albicans & Strong \\
\hline C. glabrata & Moderate \\
\hline C. Krusei & Strong \\
\hline
\end{tabular}

\subsection{Minimum Inhibitory Concentration of NSAIDs}

Sodium Diclofenac showed a higher activity and lower MIC against C. albicans and C. glabrata while Ibuprofen showed the higher activity against $C$. krusei. (Table 3 ).

Table 3. Minimum inhibitory concentrations of Ketoprofen, Ibuprofen and Sodium Diclofenac against the tested Candida spp.

\begin{tabular}{|c|c|c|c|}
\hline \multirow{2}{*}{$\begin{array}{c}\text { C. albicans ATCC } \\
\text { 10231 }\end{array}$} & Ketoprofen & Ibuprofen & Diclofenac sod \\
\hline C. albicans & 1577.5 & 500 & 52.6 \\
\hline C. glaberta & 1642.5 & 500 & 166.5 \\
\hline C. Krusi & 1045.7 & 250 & 147.6 \\
\hline
\end{tabular}

\subsection{Effect of the Tested NSAIDs on Candida spp. Membranes}

Figure 1 showed the effect of Sodium Diclofenac on Candida spp. membranes. At low concentration, Pores were formed on Candida membranes (Figure 1a). At higher concentration, complete lysis and a release to the contents of cells was observed (Figure 1b).

\subsection{Effect of NSAIDs on the Dimorphic Transition of $C$. albicans}

By testing the effect of Diclofenac sodium, Ibuprofen and ketoprofen on yeast to hyphae transition of $C$. 
albicans, it was found that Diclofenac at $500 \mu \mathrm{g} / \mathrm{ml}$ inhibited germ tube formation while Ibuprofen and ketoprofen inhibited their formation to lesser extent in all tested concentrations (Figure 2).

\subsection{Effect of Diclofenac Sodium, Ibuprofen and Ketoprofen on the Adherence And Mature Biofilms}

The effect of the tested NSAIDs on adherence and mature biofilms was found to be concentration dependent $(\mathrm{P}<0.05)$. At MIC, Sodium Diclofenac showed the highest inhibitory activity against the adherence of $C$. albicans and $C$. glabrata but Ibuprofen showed the highest inhibitory effect on the adherence of $C$. krusei. On mature biofilms, Sodium Diclofenac showed the highest activity against the tested Candida spp (Figure 3, Figure 4). At 2X MIC the same results were obtained (Table 4, Table 5).

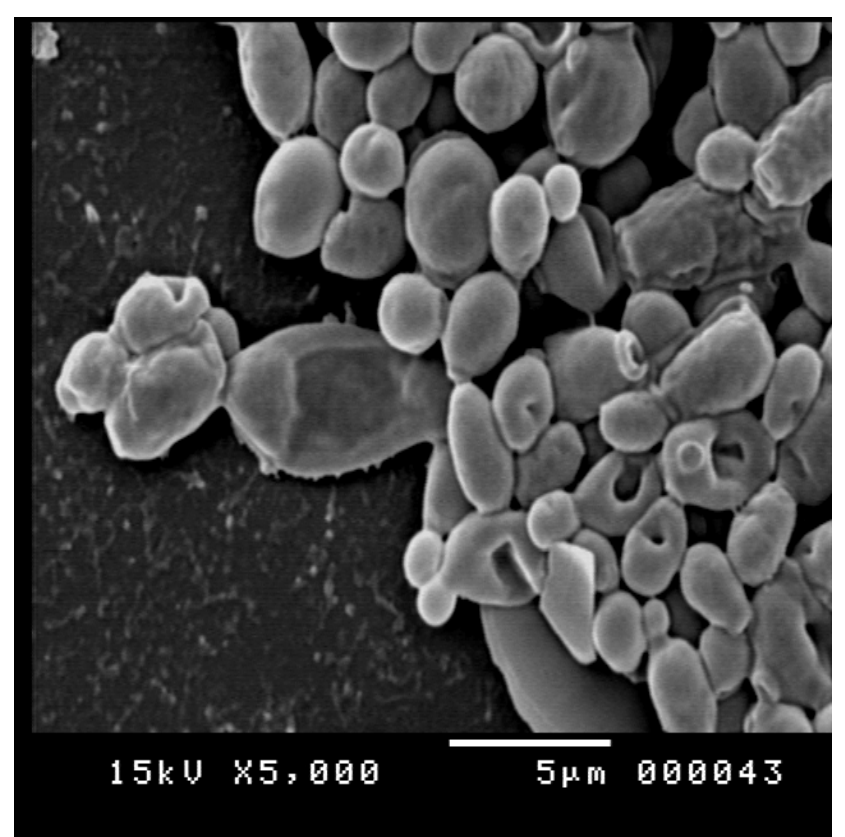

Figure 1a. At low concentration, pores were formed on the membranes of Candida cells

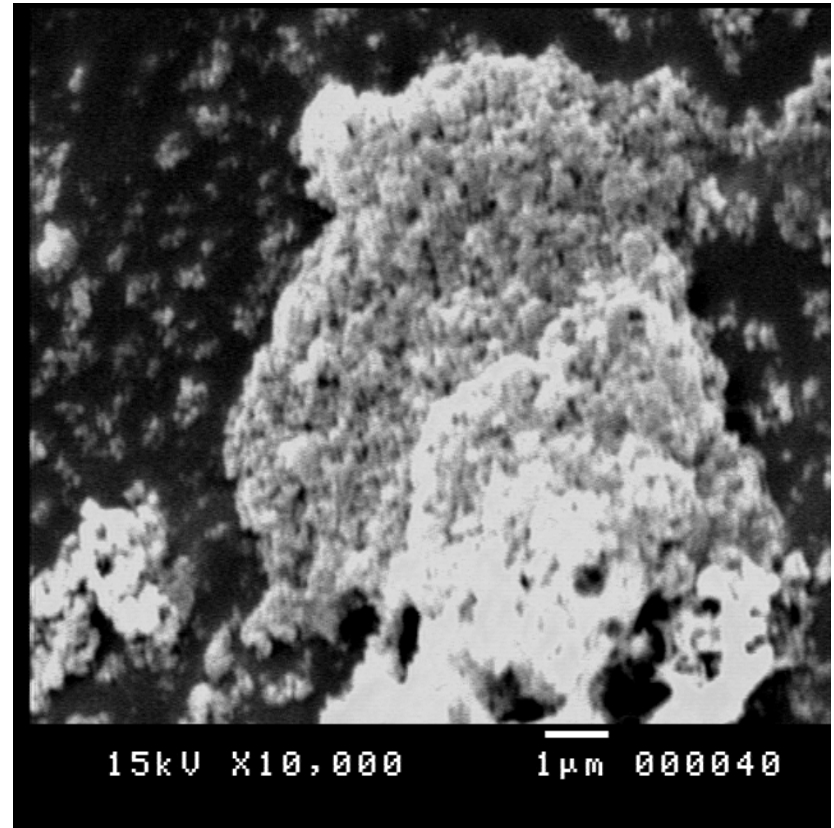

Figure 1b. complete lysis to cells were observed at high concentrations

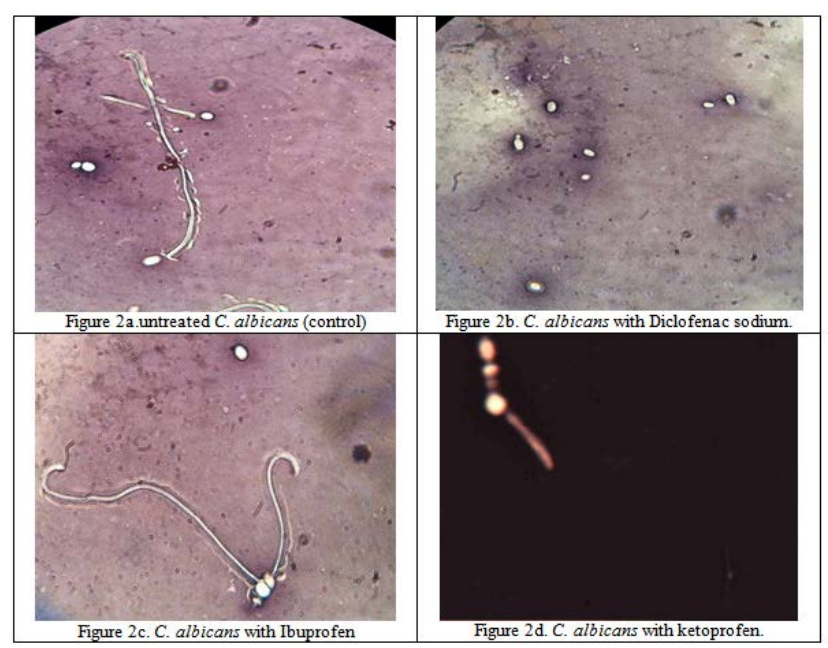

Figure 2. Effect of the tested NSAIDs on germ tube formation by $C$. albicans.

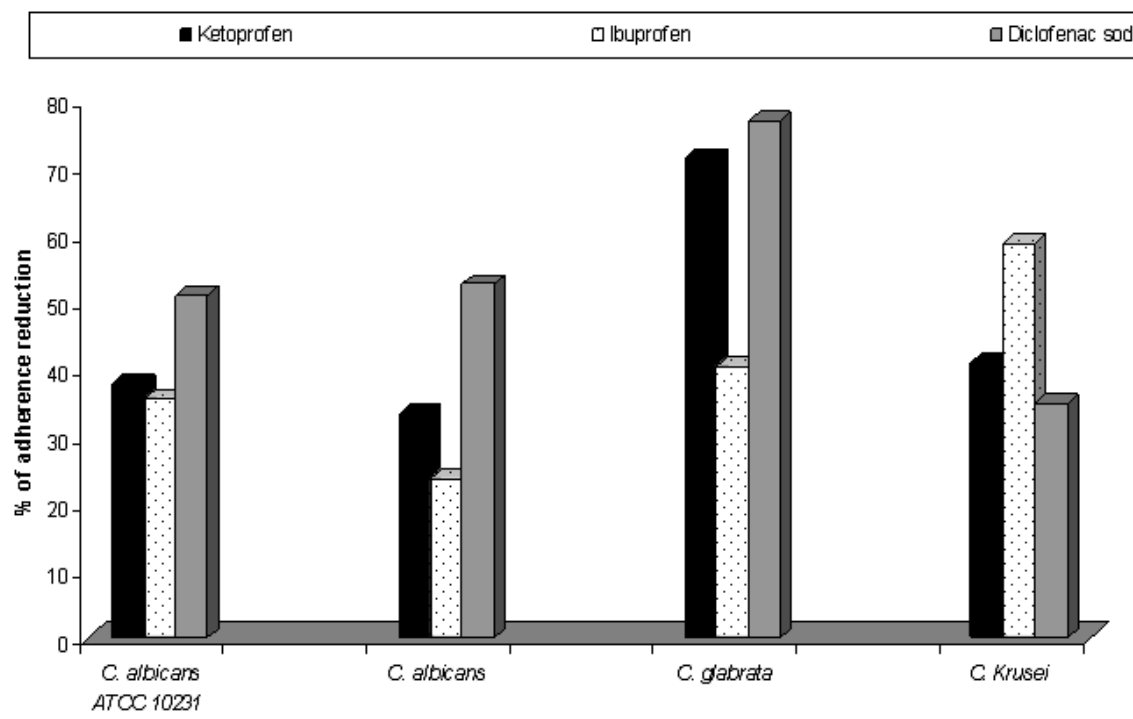

Figure 3. Effect of minimuminhibitory concentration of ketoprofen, lbupofen and diclofenac sod on the adherence of Candida spp. to the tissue culture plate 


\begin{tabular}{|c|c|c|}
\hline - Ketoprofen & Dlbuprofen & 口Diclofenac sod \\
\hline
\end{tabular}

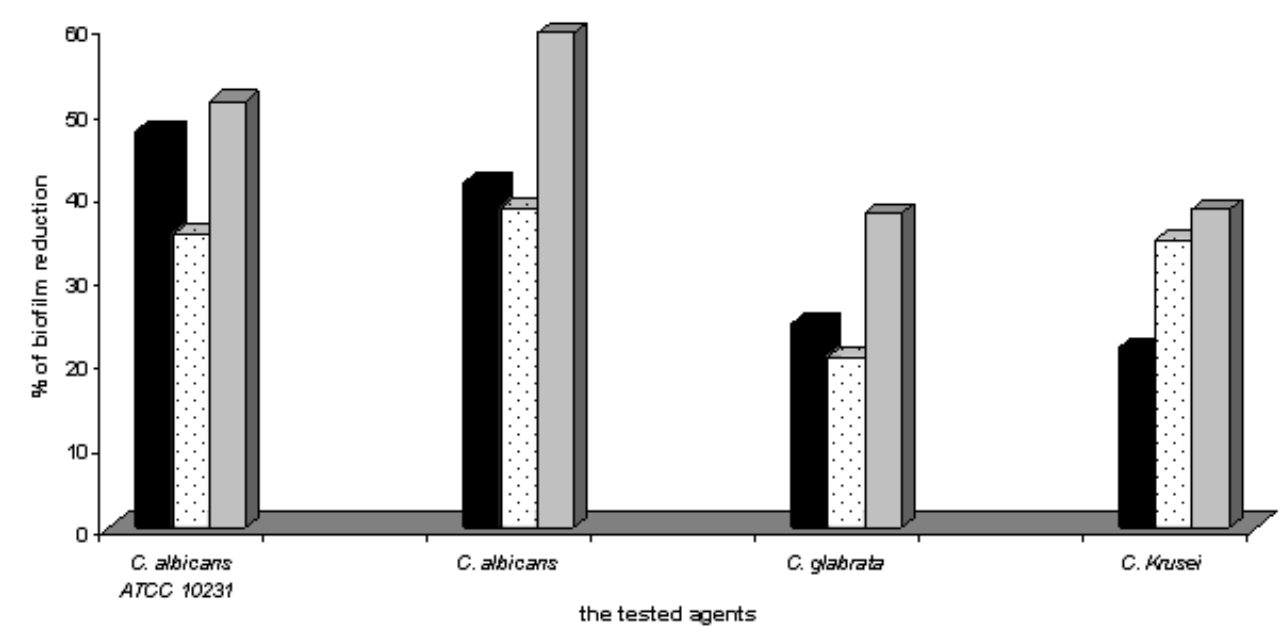

Figure 4. Effect of minimuminhibitory concentration of ketoprofen, lbupofen and diclofenac sod on mature biofilms formed by the tested Candida spp.

Table 4. Effect of MIC, Sub-MIC and above MIC concentrations on the adherence of the tested Candida spp.

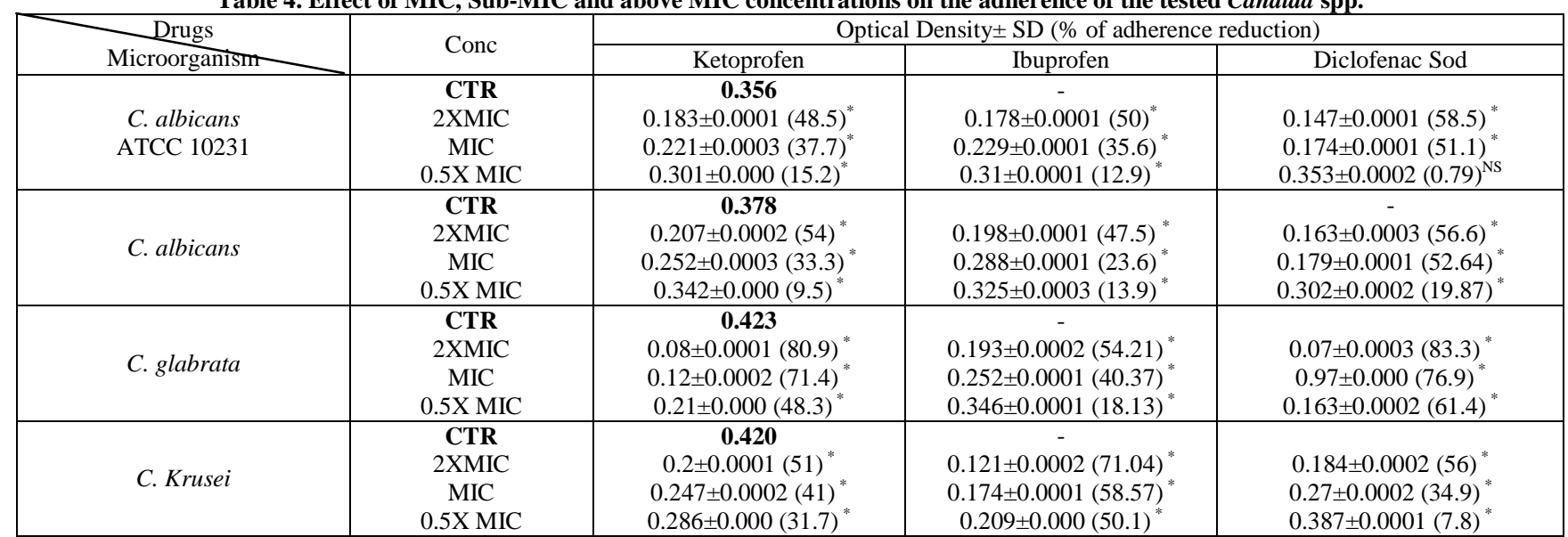

CTR: without drug (control).* $\mathrm{P}<0.05$ : Significant value, compared to controls. NS: Not significant. MIC: Minimum inhibitory concentration.

Table 5. Effect of MIC, Sub-MIC and above MIC concentrations on mature biofilms formed by the tested Candida spp.

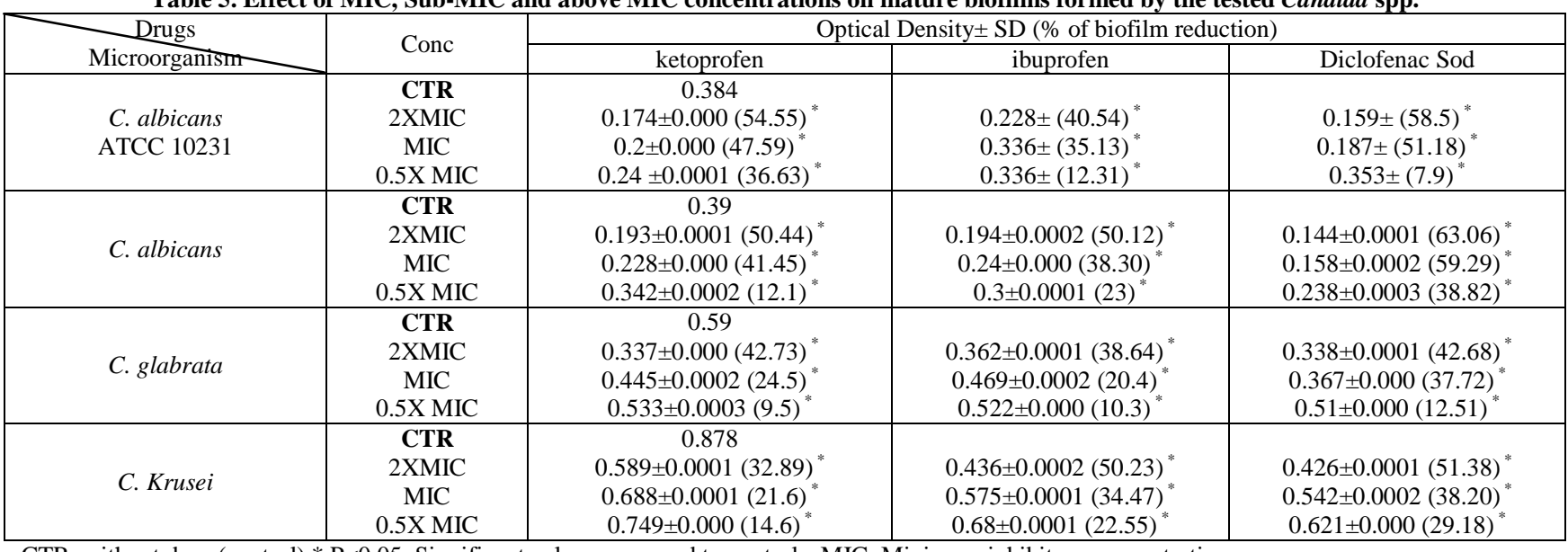

CTR: without drug (control). ${ }^{*} \mathrm{P}<0.05$ : Significant value, compared to controls. MIC: Minimum inhibitory concentration.

\section{Discussion}

Candida spp. can cause opportunistic diseases specially in patients who are immune compromised, aged, receiving prolonged antibacterial and aggressive cancer chemotherapy or undergoing invasive surgical procedures and organ transplantation. Among Candida spp., C. albicans is the most common cause of many fungal infections, but non-albicans spp. infections are in increase [33,34].

All Candida species can cause urinary tract infections (UTIs) but according to many epidemiological studies C. albicans is the most common isolated species [35,36]. Non Candida albicans also can cause fungal UTIs, and in many parts worldwide, non albicans predominate. The 
increase of antifungal resistance increase the need for new antifungals. Many studies showed that NSAID showed activity against Candida [37,38,39] that led us to undergo this study on the activity of three non-steroidal antiinflammatory drugs on $C$. albicans and 2 non-albicans strains

In this study we determined the ability of the tested organisms to form biofilm, antifungal activity of the tested drugs and their effects on the morphological switch of the tested $C$. albicans strains. The tested strains were moderate to strong biofilm producers. Also, the effects of the tested agents were found to be concentration dependent. Sodium Diclofenacshowed lower MIC against C. albicans and C. glabrata while ibuprofen showed lower MIC against $C$. krusei in comparison to other NSAIDs. By testing their effect on germ tube formation, it was found that Sodium Diclofenacat $500 \mu \mathrm{g} / \mathrm{ml}$ inhibit germ tube formation while ketoprofen and ibuprofen inhibit it to lesser extent that agree with many studies $[40,41,42]$.

In addition, The data demonstrated in this study reveal successful reduction in the in-vitro adherence and a significant destructive effect on mature biofilms of Candida spp.. Sodium Diclofenacshowed the highest inhibitory effect on the adherence of C. albicans and $C$. glabrata (51.1-76.9\% at MIC and 56.6-83.3\% at 2XMIC) and the highest disruptive effect (37.72-59.29\% at MIC and $42.68-63.06 \%$ at $2 \mathrm{XMIC}$ ) on mature biofilms formed by all the tested spp. in comparison to ibuprofen and ketoprofen. Also, by testing its effect on biofilm formed on polyurethane segments, it was found that no biofilm mass formed and the formation of pores in the membranes of Candida spp. followed by complete lysis with higher concentration of diclofenac sod.

Ghalehnoo et al., [41] and Bink et al., [42] have documented the effect of diclofenac on $C$. albicans biofilms or on cells grown on solid media. The second study [42] showed inhibition of the yeast-to-hyphae transition by diclofenac on solid YPD media. The first study demonstrated a significant reduction of biofilm formation in YPD on small polyvinyl chloride disks by diclofenac when added during the period of adhesion and biofilm formation, quantified by XTT measurements.

Alem and Douglas [43] attributed the antifungal and antibiofilm activity to the diclofenac-induced increase of the membrane permeability of the C. albicans biofilm cells which may be a result of the effect of diclofenac on the biosynthesis of fungal prostaglandins. Also, they demonstrated that biofilms of various $C$. albicans isolates and strains, grown in the presence of diclofenac, showed increased susceptibility to caspofungin. Even the activity of caspofungin against biofilms of a caspofungin-resistant isolates could be increased by diclofenac pretreatment.

Our study showed a marked activity of Ibuprofen against the tested Candida spp. especially C. krusei that agree with several studies [38,44]. The mechanism proposed for fungicidal activity of ibuprofen is in conformity with its lipophilic character [45]. Fungicidal activity of Ibuprofen is concentration dependent as it showed fungicidal activity with high doses but fungistatic activity with low doses. High doses leads to the direct damage of membranes similar to that shown by SEM images in our study. Pina-Vaz et al., [43] showed that ibuprofen at low concentration inhibit growth in a manner resembles that shown by lipophilic azoles by interfering with the metabolic pathways more than by direct damage to membranes. Also, The concentration of ibuprofen in urine of patients under the conventional standard systemic regimens reaches values above those shown to possess an antifungal activity, pointing to the potential usefulness of ibuprofen for urinary tract candidosis.

In addition, our results showed that $C$. krusei produced extensive biofilm mass on surfaces. This may be due to that C. krusei strains are more hydrophobic than C. albicans, C. tropicalis, C. kefyr, C. parapsilosis, C. glabrata or C. lipolytica isolates. C. krusei is endowed with a greater ability to colonize inert surfaces such as implants and catheters by virtue of its cell surface hydrophobicity [46].

Ketoprofen antifungal activity, their ability to decrease adherence and to detach mature biofilms were also proven by Chowdhury and Mukhopadhyay [45] and Abdelmegeed and Shaaban [47].

\section{Conclusion}

Diclofenac sodium, Ibuprofen and Ketoprofen showed antifungal activity, antiadherent activity, and can detach mature biofilms. Also, Sodium Diclofenaccan inhibit morphological switch of $C$. albicans at a concentration of $500 \mu \mathrm{g} / \mathrm{ml}$. So, these drugs are suggested to show a useful role in the treatment or the prevention of biofilm associated diseases and prevent adherence or disrupt mature biofilms of Candida Spp. to medical device surfaces.

\section{Acknowledgment}

This study was supported by Supporting of Excellence Students (SESP) Projects, Egyptian Ministry of Higher Education, Egypt.

\section{References}

[1] Douglas JL, Cobbs CG. Prosthetic valve endocarditis. In: Kaye D, Ed. Infective endocarditis. 2d ed. New York: Raven Press 1992; 375-96.

[2] Tunney MM, Patrick S, Curran MD, et al. Detection of prosthetic joint biofilm infection using immunological and molecular techniques. Methods Enzymol 1999; 310: 566-76.

[3] Raad I, Costerton W, Sabharwal U, Sacilowski M, Anaissie E, Bodey GP. Ultrastructural analysis of indwelling vascular catheters: a quantitative relationship between luminal colonization and duration of placement. J Infect Dis 1993; 168 (2): 400-7.

[4] Ramage G, Martínez JP, López-Ribot JL. Candida biofilms on implanted biomaterials: a clinically significant problem. FEMS Yeast Res 2006; 6 (7): 979-86.

[5] Seneviratne CJ, Jin L, Samaranayake LP. Biofilm lifestyle of Candida: a mini review. Oral Dis 2008, 14 (7): 582-90.

[6] LaFleur M, Kumamoto CA and Lewis K. Candida albicans biofilms produce antifungal-tolerant persister cells. Antimicrob Agents Chemother 2006, 50: 3839-46.

[7] Characklis W G, and Wilderer P A. Structure and function of biofilms. Wiley, Chichester, United Kingdom. 1989.

[8] Costerton, J. W., K. J. Cheng, G. G. Geesey, T. I. Ladd, J. C. Nickel, M. Dasgupta, and T. J. Marrie. Bacterial biofilms in nature and disease. Annu. Rev. Microbiol. 1987, 41: 435-464.

[9] Dougherty S H. Pathobiology of infection in prosthetic devices. Rev. Infect. Dis. 1988, 10: 1102-1117. 
[10] Elliott T S J. Intravascular device infections. J. Med. Microbiol. 1988, 27: 161-167.

[11] Goldmann D A, and Pier G B. Pathogenesis of infections related to intravascular catheterization. Clin. Microbiol. Rev. 1993, 6: 176-192.

[12] Gristina AG. Biomaterial centered infection: microbial adhesion versus tissue integration. Science 1987, 237: 1588-1595.

[13] Melo L F, Bott T R, Fletcher M, and Capdeville B. Biofilmsscience and technology. Kluwer Academic Publishers, Dordrecht, The Netherlands 1992.

[14] Brown M R W, and Gilbert P. Sensitivity of biofilms to antimicrobial agents. J Appl Bacteriol Symp 1993, 74:87S-97S.

[15] Cox G M, and Perfect J R. Fungal infections. Curr Opin Infect Dis 1993, 6: 422-426.

[16] Wey S B, Mori M, and Pfaller M A. Hospital-acquired candidemia. The attributable mortality and excess length of stay. Arch Intern Med 1988, 148: 2642-2645.

[17] Maki D G. Pathogenesis, prevention and management of infections due to intravascular devices used for infusion therapy, 1989, 161-177. In A. L. Bisno and F. A. Waldvogel (ed.), Infections associated with indwelling medical devices. American Society for Microbiology, Washington, D.C.

[18] Odds FC. Candida and candidosis, 2nd ed. Bailliere Tindall, London. 1988

[19] Alem MA, Douglas LJ. Effects of aspirin and other nonsteroidal anti-inflammatory drugs on biofilms and planktonic cells of Candida albicans. Antimicrob Agents Chemother 2004, 48: 41-7.

[20] Dannhardt G, Kiefer W. Cyclooxygenase inhibitors-current status and future prospects. Eur J Med Chem 2001, 36: 109-26.

[21] Alem MA, Douglas LJ. Prostaglandin production during growth of Candida albicans biofilms. J Med Microbiol 2005, 54: 1001-5.

[22] Erb-Downward JR, Noverr MC. Characterization of prostaglandin E2 production by Candida albicans. Infect Immun 2007, 75: 3498505.

[23] Pina-Vaz C, Rodrigues AG, Costa-de-Oliveira S, Ricardo E and $\mathrm{Ma}^{\circ}$ rdh P. Potent synergic effect between ibuprofen and azoles on Candida resulting from blockade of efflux pumps as determined by FUN-1 staining and flow cytometry. $J$ Antimicrob Chemother 2005 56: 678-685.

[24] Benson HC. Microbiological Application: Laboratory Manual in General Microbiology, 11th ed., McGram-Hill Higher Education, Sanfrancisco, 2002, pp.168.

[25] Christensen GD, Simpson WA, Younger J A, Baddour L M, Barrett F F, Melton D M, et al. Adherence of coagulase negative Staphylococci to plastic tissue cultures: a quantitative model for the adherence of Staphylococci to medical devices. J Clin Microbiol 1985, 22: 996-1006.

[26] O'Toole AG and Kolter R. Initiation of biofilm formation in Pseudomonas fluorescence WCS365 proceeds via multiple, convergent signaling pathways: a genetic analysis. Molecular microbiology 1998, 28: 449.

[27] Bennet JV, Brodie JL, Benner JL, Kirby WMM. "Simplified accurate method for antibiotic assay of clinical specimens". Applied Microbiology 1966, 14: 2170-2177.

[28] Negero Gemeda, Kelbessa Urga, Ashenif Tadele, Hirut Lemma, Daniel Melaku, Kissi Mudie. "Antimicrobial Activity of Topical Formulation Containing Eugenia caryophyllata L. (Krunfud) and Myritus communis L. (Ades) Essential Oils on Selected Skin Disease Causing Microorganisms.” Ethiop. J. Health Sci. 2008, 18(3): 101-107.
[29] Esimone C O, Adiukwu M U, Okonta J M, "Preliminary Antimicrobial Screening of the Ethanolic Extract from the Lichen Usnea subfloridans L,”. IJPRD 1998, 3: 99-102.

[30] Liu H, Kohler J, Fink GR. Suppression of hyphal formation in Candida albicans by mutation of a STE12 homolog. Science (New York, NY) 1994, 266: 1723-6.

[31] Xiaogang L, Zhun Y and Jianping X. Quantitative variation of biofilms among strains in natural populations of Candida albicans. Microbiology 2003, 149: 353-362.

[32] Soboh F, Khoury AE, Zamboni AC, Davidson D, Mittelman MW. Effects of ciprofloxacin and protamine sulfate combinations against catheter-associated Pseudomonas aeruginosa biofilms. Antimicrob. Agents Chemother 1995, 39: 1281-1286.

[33] Jacqueline M, Achkar, Bettina C. Candidal Infections of the Genitourinary tract. Clin. Microbiol. Rev. 2010, 23(2):253-72.

[34] Mohandas V, Ballal M. Distribution of Candida Species in Different Clinical Samples and Their Virulence: Biofilm Formation, Proteinase and Phospholipase Production: A Study on Hospitalized Patients in Southern India. J. Glob. Infect. Dis. 2011, 3 (1): 4-8.

[35] Seneviratne CJ, Jin L, Samaranayeke LP. Biofilm lifestyle of Candida: A mini review. Oral. Dis. 2008, 14: 582-590.

[36] Aparna MS, Yadav S. Biofilms: Microbes and Disease. The Braz J. Infect. Dis. 2008, 12: 526-530.

[37] Cederlund H, Màrdh P-A. Antibacterial activities of Nacetylcysteine and some non steroidal anti-inflammatory druga. $J$. Antimicrob chemother 1993 32: 903-904.

[38] Sanyal AK, Roy D, Chowdhury B, Banerjee AB. Ibuprofen, a unique anti-inflammatory compound with antifungal a activity against dermatophytes. Lett Appl Microbial 1993, 109-111.

[39] Scott E M, Tariq VN, McCrory R M. Demonsteration of synergy with fluconazole and either ibuprofen, sodium salicylate or propylparaben against C. albicans in-vitro. Antimicrob. Agents Chemother 1995, 39: 2610-2614.

[40] Alem MA, Douglas LJ. Effects of aspirin and other nonsteroidal anti-inflammatory drugs on biofilms and planktonic cells of Candida albicans. Antimicrob Agents Chemother 2004, 48:41-7.

[41] Ghalehnoo Z R, Rashki A, Najimi M, Dominguez A. The role of Sodium Diclofenacin the dimorphic transition in Candida albicans Microbial Pathogenesis 2010, 48: 110-115

[42] Bink, A, Kucharíková S, Neirinck B, Vleugels J, Dijck P V, Cammue B P. A. and Thevissen K. The Non-steroidal Antiinflammatory Drug Diclofenac Potentiates the In Vivo Activity of Caspofungin Against Candida albicans Biofilms JID 2012:206.

[43] Alem MA, Douglas LJ. Prostaglandin production during growth of Candida albicans biofilms. J Med Microbiol 2005, 54:1001-5.

[44] Pina-Vaz C, Sansonetty F, Rodrigues A et al. Antifungal activity of ibuprofen alone and in combination with fluconazole against Candida species. J Med Microbiol 2000, 49: 831-40.

[45] Chowdhury B, Mukhopadhyay S. Ketoprofen, a non-steroidal, anti-inflammatory drug, has antimicrobial activity against dermatophytes Medical Science Research. 1997, 25: 109-110.

[46] Samaranayake Y H, Samaranayake LP. Candida krusei: biology, epidemiology, Pathogenicity and clinical manifestations of an emerging pathogen. J. Med. Microbiol. 1994, 41. 295-310.

[47] Abdelmegeed E and Shaaban M I, Cyclooxygenase inhibitors reduce biofilm formation and yeast-hypha conversion of fluconazole resistant Candida albicans. J microbiol 2013, 51: 598604. 